\title{
Development of Plasma Torch - A Case Report
}

\author{
Manna S1, Sadhukhan AK ${ }^{1}$, Gupta $\mathrm{pp}^{1}$ and Chatterjee $\mathrm{PK}^{2 *}$ \\ ${ }^{1}$ Department of Chemical Engineering, National Institute of Technology, India \\ ${ }^{2}$ CSIR Central Mechanical Engineering Research Institute, India
}

*Corresponding author: Pradip Kumar Chatterjee, Central Mechanical Engineering
Case Report
Volume 2 Issue 3
Received Date: May 22, 2018
Published Date: June 13, 2018
DOI: $10.23880 /$ eoij-16000154

Research Institute (CSIR), Durgapur-713209, India, Tel: 91-343-6510236; Email: pradipcmeri@gmail.com

\section{Abstract}

This paper provides a case study of the design aspect of non-transferred and transferred torches. Designing a plasma torch, especially for the disposal of Municipal Solid Waste requires many parameters to be taken into account. The parameters are choice of material of construction of electrodes, cooling of electrodes, reduction of disintegration or consumption of electrodes, choice of carrier gas, cooling effect of carrier gas, rotation of point of discharge of electron on cathode using magnetic field, and none the less, type of power supply, that is AC, DC and Radio frequency.

Keywords: Torch; Waste to Energy; Environment

\section{Introduction}

The development of waste-to-energy plants has increased over the last 30 years as a result of the need to dispose of growing amounts of solid waste and a need to find sustainable energy sources .In the U.S., there are 87 WtE (Waste to Energy) plants handling up to 30 million tons of waste each year converting it into approximately 15 billion $\mathrm{kWh}$ of energy [1]. In India this amounts to nearly 60 million tons per day [2]. However there is only few WtE plant in operation. All of the WtE plants are based on incineration. Incineration produces carcinogenic gases and thus many of the plants have been closed down [3-5].

Although plasma gasification has higher energy input requirements than other WtE means, this technology offers advantages in waste disposal, especially the treatment of hazardous wastes, energy products recovered that offer flexibility to serve energy needs and less impact on the environment [1]. Hence an efficient plasma torch can be the answer to all the problems being faced due to arising solid waste disposal system.

Plasma torch is a device, where a jet of plasma is generated. Plasma is a collection of charged particles containing almost equal numbers of electron, positive ions in their radical state. The charged radicals are at a very high energy state. A Plasma torch comprises of anode, cathode and duct for the flow of carrier gas trough the arc established between the electrodes. The gas passing through the arc ionizes and produces plasma plume. The arc between the electrodes can be produced using DC, AC or Radio Frequency power source. The potential difference between the electrodes can vary from few volts to few Kilo Volts and the frequency from Zero (DC) to Few MHz.

There are two types of Plasma torch, transferred and Non-transferred. In the transferred torch the electrodes are separate and that in non-transferred the electrodes are housed in a common housing. For different type of usage of plasma, different types of torches are used. 


\section{Ergonomics International Journal}

\section{Design Parameters}

The most serious problem of solid electrode plasma torch is the electrode erosion. Particularly in case of high power plasma torch equipped with thermionic cathode like tungsten insufficient life time of the cathode been the most essential and urgent issue to be solved [5]. The cathode can be cooled by the carrier gas itself. The cooling effect is better with the vortex flow of the carrier gas and rotation of the electron ejection point by using magnetic field. The anode takes the impact of electron and is subjected to maximum erosion. A good cooling of anode will retard the erosion of anode. Similarly, presence of Oxygen in the carrier gas also erodes away the electrodes especially the metallic ones.

Catering to the above problem cooling of anode and cathode is also very important. The design of the torch incorporates proper flow of water extracting away the heat from the hot parts of the electrodes.

A design of a non transferred torch is as shown in the figure 1. The torch comprise of copper cathode and copper anode. Cathode is having a shape of conical nozzle to facilitate of laminar flow of carrier gas. The anode is cylindrical with co axial port for the plume to move outward through it. The anode is jacketed by stainless steel water cooled system. Cathode tip is screwed type for easy replacement in case of erosion. Anode and cathode is separated by ceramic block. The ceramic block also has the provision of anchoring both anode and cathode with it so that it is an integrated assembly. The front flange with the anode cooling jacket is used for fixing on the plasma gasifier hearth. It also provides the port for the inlet and outlet of the water for cooling. The cathode jacket has the port for carrier gas through the surface of the cathode. The cathode holder is having a coaxial port for the axial flow of the carrier gas. The cooling water inlet and outlet for the cathode jacket are taken from the rear side. A bearing based gap adjustment assemble is fitted at the rear end of the torch. This adjusts the gap between the cathode and the anode.

The torch is having a dimension of $200 \mathrm{~mm} \times 250 \mathrm{~mm}$. the power source to the torch is $80 \mathrm{~V}$ DC with current capacity of 300A. A high voltage high frequency source is superimposed with the low DC source for initial arc establishment. This arc is established without physical contact between the electrodes. This reduces the erosion of the cathode and anode and prevents sticking of the electrodes as well. The current during initial arcing is $100 \mathrm{~mA}$. After initial arcing the carrier gas gets ionized around the proximity of anode and cathode tip. This increases the conductivity in this zone and facilitates low
DC power to establish arc with full power at $300 \mathrm{~A}$. the carrier gas is then increased to get the plume out of the anode. Water circulation is established before the start up. A flow switch is must to interlock the power source with the flow of water.

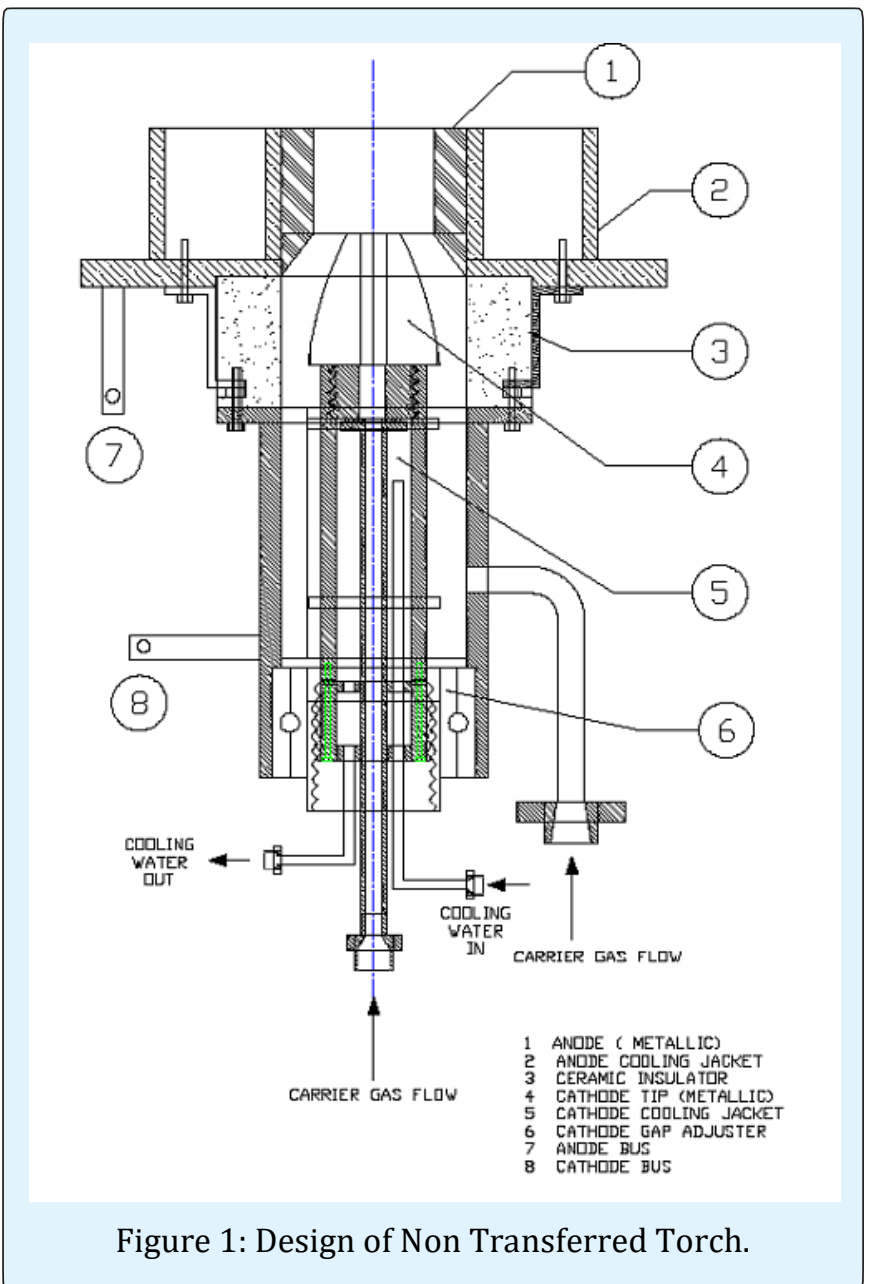

\section{Conclusion}

Metal electrodes with cooling system will enhance the longevity of the electrodes. Proper design with smaller replaceable parts will further reduce the operation and maintenance cost. A lean inert gas lamination over the cathode will further reduce the oxidation effect, in case of presence of oxygen in the carrier gas.

\section{References}

1. Larry Gray (2014) MANE 6960-Solid and Hazardous Waste Prevention and Control Engineering, Rensselaer Hartford, Hartford, USA. 


\section{Ergonomics International Journal}

2. Central Pollution Control Board (2016) Status of Compliance by CPCB with Municipal Solid Wastes (Management and Handling) Rule, 2000.

3. (2006) Incinerators in Disguise, Case Studies of Gasification, Pyrolysis, and Plasma inEurope, Asia, and the United States. Greenaction for Health and Environmental justice.
4. (2006) Global Alliance for Incinerator Alternatives (GAIA).

5. Masao Ushio (1992) Plasma application in the steel industry in Japan. Pure \& Appl Chern 64(5): 677-683. 